\title{
Microbial Production and Characterization of 3, 4- dihydroxy-L Phenylalanine Mediated an Extracellular Heterogenic Eumelanin Pigment From Marine Actinomycetes Prauserella Sp. MAPPL 017A
}

\section{Perumal Palani ( $\sim$ palani7@unom.ac.in )}

University of Madras

Chinnadurai Gandhi Shree

University of Madras

\section{Research Article}

Keywords: Extracellular, Melanin, Extraction, Purification, Characterization

Posted Date: July 14th, 2021

DOI: https://doi.org/10.21203/rs.3.rs-706088/v1

License: (c) (1) This work is licensed under a Creative Commons Attribution 4.0 International License. Read Full License 


\section{Abstract}

Melanin is one of the natural biological pigment and it is derived from the enzyme tyrosinase by the oxidation of an amino acid tyrosine. The enzyme tyrosinase [EC 1.14.18.1] is a type-3 copper protein family found large amount in microorganisms and human beings involved in the biosynthesis of melanin and other polyphenolic compounds. Melanin plays an important role in all living creatures such as photo protectants against UV radiation, antioxidant, metal adsorption, electrical conductivity, camouflage, antiaging properties, virulence and fungal pathogenesis. The above properties of melanin can be used in various application purposes which make the melanin expensive and great demand in the industry. To overcome the above situation, there is a necessity to find out the new biological sources for the maximum production of melanin. Numerous reports are available on fungal and bacterial melanin but only a few of them from actinomycetes. Based on the above view, the present investigation was aimed to purify the DOPA melanin from the novel marine actinomycetes Prauserella sp. (MAPPL 017A). Purified melanin was characterized using several state-of-the-art techniques and it's showed the broadband absorbance, presence of carboxylic and indolic groups, high amount of carbon, nitrogen and low level of sulfur, presence of other metal ions such as calcium, sodium and magnesium with efficient thermal properties.

\section{Introduction}

Melanin pigments are highly insoluble natural biological macromolecules [1] which occurs in all the animal kingdom. Based on the color and substrate sp.ecificity these macromolecules are classified into five major types such as eumelanin, pheomelanin, pyomelanin, allomelanin and neuromelanin. Among these, eumelanin is the predominant pigment synthesized in humans and microorganisms [2] which are hydrophobic, negatively charged and high molecular weight polymer [3].

In microorganisms, the melanin pigments are metabolically derived from the enzyme tyrosinase which catalyzed by the oxidation of an amino acid tyrosine [4-5] and the resultant melanin pigments are black or brown in color [3]. It is also been proved that the microbial tyrosinases are resemblance to the type-3 copper metalloprotein [6].

Melanin pigments are polymers of phenolic compounds [7] possess antioxidant and radioprotective properties which can be used as a protective shield from the harmful radiations and it is evident that the low level of melanin in the human skin has high risk of tumor [8-9]. It also plays an important role in the virulence of a broad range of phytopathogenic fungi $[3,10]$. In addition, melanin has an affinity towards the metal ions and is naturally associated with them. The above characteristics made the researchers make use of it in different technologies such as metal adsorption and electric conductivity (semiconductors) and bioelectronics. Due to the above information, the present study was aimed to purify the extracellular melanin from the novel marine Actinomycetes Prauserella sp. and this is the first report on melanin production from the genus Prauserella.

\section{Materials And Methods}




\subsection{Isolation and identification of melanin producing marine actinomycetes}

Melanin producing marine actinobacterium was isolated from water collected in the marina beech, (Lat. $13^{\circ} 3^{\prime} 20^{\prime \prime} \mathrm{N}$ and Long. $80^{\circ} 17^{\prime} 1^{\prime \prime} \mathrm{E}$ ) a region of Bay of Bengal, Chennai, Tamil Nadu, India and screened for the melanin production. The melanin positive strain was identified through polyphasic, molecular and chemotaxonomic characterization.

\subsection{Test for melanin formation and tyrosinase activity}

The test for melanin formation and tyrosinase activity were carried out by a simple, rapid and sensitive method proposed by Mikarni et al., [11].

\subsection{Extraction and purification of melanin pigment}

The extraction and purification of extracellular melanin produced by Prauserella sp. (MAPPL 017A) was performed by the method [12].

\subsection{Purification of melanin from Prauserella sp. (MAPPL 017A)}

For the purification of melanin pigments, different solvents and extraction procedures were carried out to remove the associated components such as protein carbohydrate in the melanin pigment. The removal of carbohydrates and proteins were carried out by acid hydrolysis which was followed by lipids extraction with the solvent chloroform and the metabolites were sequentially removed by the extraction of different solvents such as hexane, ethyl acetate, acetone and ethanol.

\subsubsection{Acid hydrolysis}

The crude melanin obtained from the above procedure was hydrolyzed with $10 \mathrm{ml}$ of $6 \mathrm{M} \mathrm{HCl}$ for $2 \mathrm{~h}$ at $100^{\circ} \mathrm{C}$ to remove carbohydrates and proteins. The non-hydrolysable residue was collected by centrifugation at 10,000 rpm for 10 min and washed with distilled water for several times and dried under desiccation and stored in airtight containers for further use.

\subsubsection{Washing with solvents}

The melanin obtained after acid hydrolysis was re-dissolved in $2 \mathrm{M} \mathrm{NaOH}$ and centrifuged at 10,000 rpm for $15 \mathrm{~min}$. The supernatant was collected and subjected to precipitation with $1 \mathrm{M} \mathrm{HCl}$ and centrifuged to obtain the pellet. The pellet was washed several times with distilled water and dried well. The nonhydrolysable melanin was re-dissolved in $5 \mathrm{ml}$ of $1 \mathrm{M} \mathrm{NaOH}$ to which $2 \mathrm{~mL}$ of chloroform and $0.2 \mathrm{~mL}$ of 1 butanol were added. The mixture was shaken for $30 \mathrm{~min}$ and centrifuged at 10,000 rpm for $10 \mathrm{~min}$. The chloroform and butanol phase was discarded and the procedure was repeated twice. The solution containing melanin was treated with organic solvent such as, hexane, ethyl acetate, acetone for the removable of secondary metabolites and finally with ethanol for the removable of water molecules bound 
with the melanin pigment. The resulting content was lyophilized and stored as 'pure melanin' for further characterization.

\subsection{Characterization of melanin pigment}

\subsubsection{Physio-chemical analysis of purified melanin}

The chemical analysis of purified melanin pigment was carried out by the modified method of [13-14]. Commercially available synthetic melanin was purchased from Sigma (Catalog No. M 8631) and used as the standard for all the characterization directly without any further treatment.

\subsubsection{UV-Vis sp.ectroscopic analysis of purified melanin}

The purified melanin was dissolved in $0.1 \mathrm{M}$ sodium carbonate buffer $(\mathrm{pH} 10.3)$ and $0.1 \mathrm{M}$ sodium hydroxide solution to obtain a final concentration of $0.03 \mathrm{~g} / \mathrm{L}$. The solutions were stirred by vortexing for $15 \mathrm{~min}$ and centrifuged at $10,000 \mathrm{rpm}$ for $15 \mathrm{~min}$ at $4^{\circ} \mathrm{C}$. The supernatant collected was subjected to UVVis sp.ectral analysis at a wavelength range of $200-800 \mathrm{~nm}$ in a UV-Vis sp.ectrophotometer (Hitachi U2900, Japan).

\subsubsection{FT-IR analysis of purified melanin}

For FT-IR analysis, approximately $5 \mathrm{mg}$ of melanin and $700 \mathrm{mg}$ of $\mathrm{KBr}$ were thoroughly mixed and made into pellets. The pellet was used for recording the IR sp.ectra using an FT-IR sp.ectrometer (Perkin Elmer, USA).

\subsubsection{Raman sp.ectroscopic analysis of purified melanin}

The purified melanin was subjected to Raman sp.ectroscopic analysis (Raman 11i, Nano photon, Japan) at the range of $1000-2500 \mathrm{~cm}^{-1}$. The sp.ectrometer has back scattering geometry for the detection of Raman sp.ectrum with the resolution of $1 \mathrm{~cm}$ and the excitation source was $532 \mathrm{~nm}$.

\subsection{5 ${ }^{1} \mathrm{H}$ NMR sp.ectral analysis of purified melanin}

The melanin pigment was dissolved in DMSO and subjected to ${ }^{1} \mathrm{H}$ NMR sp.ectral analysis with Bruker instrument (400 MHZ) at Sophisticated Analytical Instruments Facility (SAIF), Indian Institute of Science, Bangalore.

\subsubsection{Solid state NMR analysis of purified melanin}

The solid-state NMR sp.ectral analysis of purified melanin was performed with a Bruker Avance-III HD 400 WB NMR sp.ectrometer (9.4 T). The proton and carbon resonance frequencies were set at 400.07 and $100.61 \mathrm{MHz}$, resp.ectively. Samples were packed in a 4-mm-diameter zirconia rotor with a Kel-F cap. ${ }^{13} \mathrm{C}$ MAS sp.ectrum of the sample was recorded using a double resonance $4 \mathrm{~mm}$ MAS probe at room temperature. ${ }^{13} \mathrm{C}$ cross polarization/total sideband suppression (CP/TOSS) analysis was performed as 
described by Dixon [15] at a sp.inning sp.eed of $7 \mathrm{kHz}$ to get sideband free sp.ectrum. A contact time of 3 ms and a ${ }^{1} \mathrm{H} 90^{\circ}$ pulse length of $4 \mu$ s were used. Typically, 768 scans were acquired with a relaxation delay of 4s. According to Fung et al., [16] the SP.INAL-64 decoupling sequence was used to decouple protons during the carbon acquisition employing the radio frequency field strength of $83 \mathrm{kHz}$. FID was subjected to an exponential multiplication function with a line broadening value of $150 \mathrm{~Hz}$ prior to Fourier transform. ${ }^{13} \mathrm{C}$ chemical shifts were referenced to the carbonyl signal of glycine at $176.03 \mathrm{ppm}$ as an external reference standard.

\subsubsection{Quantitative elemental analysis of purified melanin.}

The percentage of elements such as carbon, hydrogen, nitrogen and sulfur present in the melanin pigment was determined over a wide range of sample matrices and concentrations with Trusp.ec micro analyzer at Sophisticated Analytical Instruments Facility, IIT Madras, Chennai, India.

\subsubsection{Inductively Coupled Plasma Optical Emission Sp.ectrometry (ICP-OES) analysis of purified melanin}

The atomic sp.ectrum emitted by purified melanin is used to determine its elemental composition by ICPOES analysis (Perkin Elmer optima 5300 DV, USA). The wavelength at which emission occurs identified the element while the intensity of the emitted radiation quantifies its concentration.

\subsubsection{Procedure for Melanin digestion for ICP-OES}

Thirty milligrams of melanin was dissolved in $10 \mathrm{~mL}$ of deionized water which was added with $1 \mathrm{~mL}$ each of nitric acid $(65 \%, \mathrm{w} / \mathrm{v})$ and perchloric acid $(70 \%, \mathrm{w} / \mathrm{v})$. The mixture was heated at $100^{\circ} \mathrm{C}$ for $5 \mathrm{~min}$ and the volume was made up to $100 \mathrm{~mL}$ with deionized water.

\subsubsection{High Resolution Scanning Electron Microscopic and Energy Disp.ersive Sp.ectrometer analyses (HRSEM-EDAX) of purified melanin}

The surface topography and qualitative elemental analysis of the purified melanin was performed using HRSEM-EDAX (FEI QUANTA FEG 200 Hillsboro, USA) equipped with EDX at Sophisticated Analytical Instruments Facility, IITM, Chennai.

\subsubsection{Electro paramagnetic resonance analysis of purified melanin}

Electron paramagnetic resonance of the purified melanin was acquired with a Varian X-band EPR sp.ectrometer (JEOL Model JES FA 200, Japan) with a modulation frequency of 8.75-9.65GHz. The gvalue of the melanin sample was measured at the point of zero slope of the absorption curve, and the line width $(\mathrm{AH})$ was measured between inflection points of the resonance absorption [17].

\subsubsection{X-ray diffraction analysis of purified melanin}

The purified melanin obtained from Prauserella sp. was ground to fine powder with mortar and pestle and subjected to analysis by $X$-ray diffraction with the following parameters: CuKa radiation $(\lambda-1.5406 \AA)$, 
voltage $40 \mathrm{kV}$, current $40 \mathrm{~mA}, 2 \theta$ range $3^{\circ}-80^{\circ}$.

\subsubsection{Thermal degradation analysis of purified melanin}

The purified melanin obtained from Prauserella sp. was weighed $(3.343 \mathrm{mg})$ and analyzed with a thermal analyzer (SDTQ600v 8.0 build 95). The TGA and DSC data were recorded under nitrogen atmosp.here at the flow rate of $20 \mathrm{~mL} / \mathrm{min}$. The analysis was carried out with ambient temperature to $1000^{\circ} \mathrm{C}$ at a heating rate of $10^{\circ} \mathrm{C} / \mathrm{min}$.

\section{Results And Discussion}

\subsection{Isolation and identification of melanin producing marine actinomycete}

Pigment producing isolate MAPPL 017A was isolated using the sea water collected from the sea shore of marina beech, a region of Bay of Bengal, Chennai, Tamil Nadu, India. And produced the diffusible purplish black color pigments on Czapek tyrosine (Fig. 1). The resultant pigment was confirmed as melanin through standard protocols. The melanin positive organism MAPPPL 017A was identified as Prauserella $\mathrm{sp}$. based on the morphological, physiological, biochemical, molecular and chemotaxonomic identification methods. The resultant nucleotide sequence from the above strain was deposited in the gene bank with the accession no MH341971.

\subsection{Melanin Confirmation test}

The qualitative and quantitative determination of melanin production by the isolate MAPPL 017A was carried out by the addition of L-DOPA to the culture supernatant. The color changes into brown confirmed the presence of enzyme tyrosinase (Fig. 2) and the amount of tyrosinase enzyme produced by the melanin positive isolate was quantitatively determined and the maximum enzyme production occurred on 15 day (Fig. 2). Similar experiments were carried out in Streptomyces griseus and Streptomyces phaeochromogenes [11].

\subsection{Purification of melanin pigments produced by Prauserella sp.}

The purification of melanin produced by Prauserella sp. was done using acid precipitation method which is the familiar and sp.ecific method preferred by most of the researchers $[12,14,18-34]$ and it was characterized using several state-of-the-art techniques which are described below.

\subsection{Physical properties of purified melanin}

The purified melanin has been found to be insoluble in water, organic solvents and partially soluble in DMSO. However, the pigment was completely soluble in ammonia water, $\mathrm{NaOH}$ and $\mathrm{KOH}$ solutions. The purified melanin has been found to be in characteristic black color, viscous in nature and susceptibility to oxidizing agents [35] as has been observed with melanin pigments from various bacterial, fungal and plant sources (Bell) [39] such as seeds of O. fragrans [14], Klebsiella sp. GSK [21], Asp.ergillus nidulans 
[22], Asp.ergillus bridgeri ICTF-201 [24], Pseudomonas stutzeri [28] Aeromonas media [37], Vibrio cholerae [38], Klebsiella pneumoniae [39], Tea leaves [18, 40], Escherichia coli [41-43], Frankia strain [44], Pleurotus cystidiosus [45] and Black-bone silky fowl $[42,43,46]$ as well as synthetic melanin.

\subsection{UV-Vis Sp.ectroscopic analysis of purified melanin}

Melanin is an amorphous pigment which naturally exhibit scattering phenomenon (broadband absorption) in the UV-Vis sp.ectrum (scattering phenomenon) and it believed to be formed by the superposition of the peaked sp.ectra which are termed as "chemical disorder model" [47-49]. The melanin pigments show strong UV absorption in the region of 200-300 nm which could be attributed to the $\pi=>\pi^{*}$ and $n=>\pi^{*}$ of the amino, carboxylic and aromatic moieties [50]. In the present study, the purified melanin pigment obtained from Prauserella sp. and synthetic melanin showed strong absorbance in the shorter wavelengths such as 211 and $213 \mathrm{~nm}$, resp.ectively (Fig. 3A) and the absorption decreased rapidly when the wavelengths increased to $400-800 \mathrm{~nm}$. The absorbance of the melanin pigments were monotonically increased towards the higher energy region (200-300 nm) as it can be observed only in the range of 210$240 \mathrm{~nm}$ and decreased rapidly with increasing wavelength towards the visible region (400-800). The above characteristics have been considered as general features of the melanin pigments [51] which increases the protection against the most damaging high energy from harmful radiations. This phenomenon is due to the superposition of absorption of individuals, distinct chemical constituents [52] and the presence of complex conjugated structure of the melanin pigments.

\subsection{FT-IR sp.ectroscopic analysis of purified melanin}

The functional groups present in the purified melanin have been characterized with FT-IR sp.ectroscopic analysis. The broad absorption band centered around 3450 and $3445 \mathrm{~cm}^{-1}$ with EM and SM, resp.ectively has been found to be characteristic of $\mathrm{O}-\mathrm{H}$ or $\mathrm{N}-\mathrm{H}$ stretching vibrations as has been observed by many investigators [24, 27, 53-57]. The strong characteristic band observed at 1637 and $1636 \mathrm{~cm}^{-1}$ in resp.ect of EM and SM have been attributed to the bending vibrations modes of aromatic ring $\mathrm{C}=\mathrm{C}$ and $\mathrm{C}=\mathrm{N}$ bond of aromatic system in addition to $\mathrm{C}=\mathrm{O}$ double bond $(\mathrm{COOH})$ of carboxylic function involved in the bond formation with the metal ions $[2,24,27,53,54,57-60]$. This may be due to the presence of carbonyl groups conjugated with a benzene ring and a similar situation has been found with quinone structure of DOPA- melanin [61]. Finally, the weak bands observed below $700 \mathrm{~cm}^{-1}$ have been ascribed to alkenes $\mathrm{C}-\mathrm{H}$ substitution and out-of-plane bending of the aromatic carbon-hydrogen bond in the melanin pigment. The signals present at the region of $600-500 \mathrm{~cm}^{-1}$ (below $700 \mathrm{~cm}^{-1}$ ) have been attributed to the out-of-plane bending of the oxygen hydrogen bonds $[24,57,60,62,63]$ and $720-590 \mathrm{~cm}^{-1}$ areas indicate the presence of aliphatic iodo group (Fig. $3 \mathrm{C}$ ). The above observation made in the present study has been found to be similar to the eumelanin obtained from Sepia officinalis $[55,64-66]$.

\subsection{Raman sp.ectroscopy analysis of purified melanin}


The molecular vibration and crystal structures of the purified melanin was analyzed using Raman sp.ectroscopic analysis. The extra cellular melanin (EM) from Prauserella sp. and Synthetic melanin (SM) exhibited two prominent peaks in the ranges between $1300 \mathrm{~cm}^{-1}-1600 \mathrm{~cm}^{-1}$ (Fig. 3B). The presence of lower wave number centered at $1345 \mathrm{~cm}^{-1}, 1340 \mathrm{~cm}^{-1}$ (EM and SM) were related to the $\mathrm{C}-\mathrm{N}$ stretching mode of the indole structure and peaks centered at $1557 \mathrm{~cm}^{-1}, 1576 \mathrm{~cm}^{-1}$ (EM and SM) were assigned to aromatic $\mathrm{C}=\mathrm{C}$ stretching modes of the basic indole structure and $\mathrm{C}=\mathrm{N}$ stretching $/ \mathrm{N}-\mathrm{H}$ bending (Matsunuma, Capozzi, 2005, Nagano, Priti Vairale) [67-70]. Similar peak were observed in cutaneous melanin [71]

\subsection{Electro paramagnetic resonance sp.ectroscopic analysis of purified melanin (EPR)}

The EPR sp.ectrum obtained with melanin pigments from Prauserella sp. appeared as a singlet without any hyperfine structure as found in synthetic melanin and DPPH, a well-known antioxidant compound (Fig. 4). EPR Sp.ectroscopy analysis of purified melanin pigment produced signals at the magnetic fields 337.918 [mT], 337.919[mT], 337.881[mT], and their G-values were calculated as 1.99776, 1.99743, 1.99751 for extracellular melanin, synthetic melanin and DPPH, resp.ectively. Similar observations were found in Cryptococcus neoformans [72], Paracoccidioides brasiliensis [73], Histoplasma capsulatum [74], Sp.orothrix schenckii [75], Sepia officinalis [75], Klesiella sp. [21]. The G-values obtained with purified melanin were found to be nearer to the value of standard antioxidant compound which indicated the presence of stable free radicals in the melanin pigments [77].

\section{$3.9^{1} \mathrm{H}$ Nuclear magnetic resonance (NMR) sp.ectral analysis of purified melanin}

The NMR sp.ectral analysis performed with purified extracellular melanin from Prauserella sp. has shown signals in both aliphatic and aromatic regions (Fig. 5A \& B). The peaks centered in the region of 0.2-2.0 ppm could be assigned to the $\mathrm{CH}_{3}$ group of alkyl fragments such as $\mathrm{CH}_{2} \mathrm{CH}_{3}, \mathrm{CH}\left(\mathrm{CH}_{3}\right)_{2}$ as has been observed with melanin pigments obtained from different organisms [30, 31, 54,60,78-79]. The peaks centered between 2.2-2.8 ppm indicated the presence of characteristic methylene group [54,60,78] while the peak centered at $3.5 \mathrm{ppm}$ has been assigned to the presence of methyl $\mathrm{N}-\mathrm{CH}_{3}$ [78] which might be attached to either the pyrrole or indole ring [80]. The peaks centered in the region of 3.5-4.2 ppm have been assigned to the carbon attached to nitrogen or carbon $\left(-\mathrm{CH}_{2}\right.$ or $-\mathrm{CH}_{3}$ group connected with $\left.\mathrm{N} / 0\right)$ as has been found in pigments of other $[30,54,60]$. The peaks observed in the region of 4.2-5.4 ppm are due to the presence of $\mathrm{C}=\mathrm{C}-\mathrm{H}$ structures in the aromatic nucleus $[54,60]$. The peaks centered between $6.5-9.0$ $\mathrm{ppm}$ have been assigned to the proton attached to the substituted aromatic and hetero-aromatic regions $[30,54,60,79]$.

\subsection{Solid state ${ }^{13} \mathrm{C}$ NMR analysis of purified melanin}

The solid state NMR analysis performed with purified melanin obtained from Prauserella sp. has shown distinctive sp.ectral regions (Fig. 6) such as aliphatic (10-110), aromatic (110-160) and carbonyl groups (160-225) which corroborates the observation made with Sepia melanin, acid free Sepia melanin and 
melanin from human hair [81]. The peaks observed in the lower region (10-95ppm) are characteristics of aliphatic carbons, which might have arisen from the proteinaceous substance as sociated with pigment as has been observed in Catharsius molossus [79] and Auricularia auricula [82]. The aliphatic carbon in the lower regions centered at 10-40 ppm could be due to the presence of the alkyl group such as ($\left.\mathrm{CH}_{2} \mathrm{CH}_{3}\right)$, methyl or $\left(-\mathrm{CH}_{2}^{-}\right)$methylene and the peaks centered in the region of $40-60 \mathrm{ppm}$ are due to the presence of a-carbon or carbon in $\mathrm{CH}-\mathrm{N} / \mathrm{CH}-\mathrm{S}$. The results obtained in the present study corroborates with the observation made earlier by [60, 82-85]. In addition, the peaks observed in the region of 35.2 and 55.7 ppm has been found to show the presence of methyl $\left(\mathrm{N}^{-} \mathrm{CH}_{3}\right)$ group [86]. The peaks observed in the region 95-160 and 110-160 ppm is characteristic of aromatic carbons which indicate the presence of indole or pyrrole types of carbons in the melanin polymers. The presence of broad signals in the aromatic region (110-160ppm) is characteristic of melanin signal [83] as has been observed with natural and synthetic melanin $[60,79,81-83,86-89]$. The peaks centered in the region 165-225 ppm are as a result of carbonyl carbon atoms derived from peptide bonds, carboxylates, amides and quinones which might be coupled with the melanin polymers or proteins. A peak observed in the region $175 \mathrm{ppm}$ is characteristic of quinine moieties of the carbonyl group of the melanin pigments which has been found in other melanin pigments $[60,79,81-83,89-90]$.

\subsection{Elemental analysis of purified melanin}

Melanin pigments naturally have the tendency to bind to various metals and therefore the CHNS analysis has been routinely performed to identify the type of melanin and to determine the purity of the pigment. The monomeric units of eumelanin and pheomelanin resp.ectively should have 6-9 and 8-11\% nitrogen content with a sulfur content of $9-12 \%$ in the case of benzothiazine monomer [91-92]. The elemental analysis performed with the purified melanin has shown the presence of relatively higher content of carbon (41.04\%) when compared to other elements such as hydrogen $(8.18 \%)$ and nitrogen $(7.15 \%$; Table. 1). However, the melanin obtained from different sources have exhibited varied elemental compositions $[18,22,31,60,79,93-99]$. The sulfur content found in the EM and SM in the present study has been 1.56 and $0.66 \%$, resp.ectively. Naturally, the subunits of eumelanin and pheomelanin are formed through similar pathways and exist in a mixed form however it can be differentiated based on the percentage of sulfur content. The sulfur content of eumelanin obtained from different sources has been found to be in the range of 0.001 to $14.83 \%[31,63,91,98-99]$. It has been suggested that the sulfur content in the melanin samples might be due to the addition of some thiol containing compounds or presence of sulfur containing aminophenol in the melano proteins during the polymerization of eumelanin.

Table 1. Quantitative elemental analysis of melanin pigments from Prauserella sp.. (MAPPL 017A) through CHNS analysis 


\begin{tabular}{|c|c|c|c|c|}
\hline S. No. & Elements & Extracellular melanin & Synthetic melanin & $\begin{array}{l}\text { Sepia melanin (Magarelli, } \\
\text { et al.,2010) }\end{array}$ \\
\hline 1. & Carbon & 41.0350 & 46.105 & 33.859 \\
\hline 2. & Hydrogen & 8.1800 & 8.6280 & 3.362 \\
\hline 3. & Nitrogen & 7.1470 & 5.3290 & 6.068 \\
\hline 4. & Sulfur & 1.5585 & 0.660 & $<0.001$ \\
\hline
\end{tabular}

3.12 Inductively Coupled Plasma Optical Emission sp.ectrometry (ICP-OES) analysis of purified melanin

The melanin pigments isolated from a sp.ecific environment has been linked with numerous metal ions including $\mathrm{Mg}$ (II), $\mathrm{Ca}$ (II), $\mathrm{Na}(\mathrm{I}), \mathrm{K}$ (I) and almost all the first transition metals have been shown to contain $\mathrm{Fe}$ (III) in abundant quantity. Therefore, melanin pigments could serve as a reservoir of metal ions such as $\mathrm{Ca}$ (II) $\mathrm{Cu}$ (II) and Fe (III). In the present investigation, the quantitative determination of the abundant metal ions in the purified melanin pigments has been carried out with ICP-OES analysis which indicated the presence of $\mathrm{Na}^{+}, \mathrm{K}^{+}, \mathrm{Mg}^{+}, \mathrm{Ca}^{+}$and $\mathrm{Fe}^{+}$. Both $\mathrm{EM}$ and $\mathrm{SM}$ have been found to contain the same set of metal ion with varied concentration (Table. 2). Similar observation has been made with melanin of Sepia officinalis [66] and Pseudomonas stutzeri [28].

Table 2. Quantitative elemental analysis of melanin pigments from Prauserella sp.. (MAPPL 017A) through ICP-OES analysis

Extra Synthetic melanin Sepia melanin

S. No. Elements Wavelength $(\mathrm{nm}) \quad$ cellular melanin

(Magarelli

et al., 2010)

Concentration $(\mathrm{mg} / \mathrm{g})$

\begin{tabular}{llllll}
\hline 1. & $\mathrm{Ca}$ & 317.933 & 1.393 & 3.789 & 47.302 \\
\hline 2. & $\mathrm{Fe}$ & 238.204 & 0.189 & 1.569 & 0.100 \\
\hline 3. & $\mathrm{K}$ & 766.490 & 0.090 & 0.140 & 1.300 \\
\hline 4. & $\mathrm{Mg}$ & 285.213 & 0.648 & 1.714 & 17.310 \\
\hline $\mathbf{5 .}$ & $\mathrm{Na}$ & 589.592 & 1.948 & 5.144 & 5.500
\end{tabular}

\subsection{HRSEM-EDAX analysis of purified melanin}

In the present study the morphological characteristics and quantitative determination of the elements present in the melanin pigments have been carried out with SEM-EDAX analysis (Fig.7 \& 8). The obtained biopolymer appeared to be in amorphous nature as has been observed in synthetic melanin as well as the melanin obtained from a mushroom, Auricularia auricula ([82], silky fowl [99] however the melanin 
pigments obtained from Jurassic period have been found to be sp.herical in shape [89]. The elemental composition in resp.ect of EM and SM has been found in the following orders $\mathrm{C}, \mathrm{O}, \mathrm{N}, \mathrm{S}, \mathrm{Cl}, \mathrm{Fe}, \mathrm{K}, \mathrm{Na}$ and $\mathrm{C}, \mathrm{O}, \mathrm{N}, \mathrm{Fe}, \mathrm{Cl}, \mathrm{S}, \mathrm{K}, \mathrm{Na}$. It has been observed that carbon, oxygen and nitrogen have been found to be the most abundant elements while sulfur, chlorine, iron, potassium and sodium are the least elements (Table. 3). A higher quantity of carbon, nitrogen and lower quantity of oxygen was observed in the purified melanin when compared to the synthetic melanin which indicated the density of the melanin layers. A traces of sulfur content was also found in the purified melanin which could be due the presence of copolymer of eumelanin and pheomelanin or aminophenol in the melanoprotein.

Table 3. Quantitative elemental analysis of extracellular melanin from Prauserella sp.. (MAPPL 017A) through EDAX analysis

\begin{tabular}{|c|c|c|c|c|c|c|}
\hline \multirow[t]{2}{*}{ S. No. } & \multirow[t]{2}{*}{ Element } & \multicolumn{2}{|c|}{ Extracellular melanin } & \multicolumn{2}{|c|}{ Synthetic melanin } & \multirow{2}{*}{$\begin{array}{l}\text { 3.14 X-Ray Diffraction Analysis (XRD) } \\
\text { of purified melanin }\end{array}$} \\
\hline & & Wt\% & At\% & $\mathbf{W t} \%$ & At\% & \\
\hline 1. & $\mathrm{CK}$ & 61.47 & 69.47 & 60.66 & 68.58 & The XRD sp.ectra of melanin \\
\hline 2. & NK & 09.12 & 08.84 & 07.19 & 06.97 & sp. has shown broad peak on the $2 \theta$ \\
\hline 3. & $\mathrm{OK}$ & 22.28 & 18.90 & 26.76 & 22.71 & scale of $20^{\circ} \mathrm{C}$ in all the diffractograms \\
\hline 4. & $\mathrm{NaK}$ & 00.08 & 00.05 & 00.22 & 00.13 & (Fig. 9). The broad band peaks \\
\hline 5. & SK & 03.46 & 01.47 & 00.58 & 00.25 & amorphous materials like melanin \\
\hline 6. & ClK & 02.81 & 01.07 & 01.64 & 00.63 & polymers appeared in the region of \\
\hline 7. & KK & 00.11 & 00.04 & 00.22 & 00.08 & $\begin{array}{l}20^{\circ} \mathrm{C} \text { which might be due to the } \\
\text { parallel planar layers found in }\end{array}$ \\
\hline 8. & FeK & 00.68 & 00.17 & 02.73 & 00.66 & melanin structure [100]. Melanin \\
\hline
\end{tabular}

molossus [79] and Pseudomonas stutzeri [28] have exhibited similar peaks.

\subsection{Electron Sp.ray lonization Mass Sp.ectroscopic (ESI-MS) analysis of purified melanin}

Due to the remarkable structural diversity and amorphous nature, melanin pigments fail to dissolve in organic solvents. Therefore, investigations to study their structural property and functions have become difficult task however are considered essential for application purposes. Therefore, the characterization of melanin pigments has been performed with Mass Sp.ectrometry (MS) such as ESI-MS and MALDI-MS [101-104]. In the present study, the ESI-MS analysis has revealed the molecular masses of $341.0523 \mathrm{~m} / \mathrm{z}$ and the molecular formula was derived as $\mathrm{C}_{18} \mathrm{H}_{10} \mathrm{~N}_{2} \mathrm{O}_{4} \mathrm{Na}$, which fell in the lower molecular mass range (Fig. 10) as has been observed earlier in LEM404 [31].

\subsection{Thermal properties of purified melanin}

\subsubsection{Thermal gravimetric analysis}


The extracellular melanin from Prauserella sp. underwent two stages of thermal degradation. The initial degradation occurred between $75.40-97.47^{\circ} \mathrm{C}$ which was treated as the glass transition temperature where the compound lost a weight of $0.394 \mathrm{mg}(11.79 \%)$ as a result of loss of free and bound water molecules present in the melanin pigment. The second degradation occurred at $236.53-369.80^{\circ} \mathrm{C}$ which resulted in the loss of $1.252 \mathrm{mg}(37.44 \%)$ due to the destruction of advanced and sub-structure of melanin pigments, non-covalent bonds present between layers of the structural units, covalent bonds of the monomer units such as indole and pyrrole group. The major weight loses occurred at 88.21 and $302.01^{\circ} \mathrm{C}$ and their derived weight were $0.1458 \mathrm{mg} / 3.74 \mathrm{~min}$ and $0.1946 \mathrm{mg} / 14.31 \mathrm{~min}$, resp.ectively. The complete weight loss occurred at $979.00^{\circ} \mathrm{C}$ (Fig. 11 A and Table. 4). Similar observations have been made for the melanin obtained from Catharsius molossus [79].

Table 4. Thermal gravimetric analysis of extracellular melanin from Prauserella sp.. (MAPPL 017A)

\begin{tabular}{|c|c|c|c|}
\hline \multirow[b]{2}{*}{ S. No. } & Temperature & Weight & Weight \\
\hline & Degree Celsius & $\mathrm{Mg}$ & $\%$ \\
\hline \multicolumn{4}{|c|}{ Step transition } \\
\hline 1. & $75.40-109.86$ & 0.3941 & 11.79 \\
\hline 2. & $236.53-369.80$ & 1.252 & 37.44 \\
\hline \multicolumn{4}{|c|}{ Residue } \\
\hline 3. & 979.00 & 0.8506 & 25.44 \\
\hline \multicolumn{4}{|c|}{ Point value } \\
\hline 4. & 88.21 & 3.74 & 0.1458 \\
\hline 5. & 302.01 & 14.31 & 0.1946 \\
\hline
\end{tabular}

\subsubsection{Differential Scanning Calorimetric analysis of purified melanin}

It was observed that there was a difference in the heat flow at three different temperature ranges such as $85.04-111.22,210.61-247.57$ and $272.79-309.80^{\circ} \mathrm{C}$ and the maximum heat energy absorption were at 91.47/0.06059 area j/g, 229.61/86.44 area j/g and 282.65/23.62 area j/g, resp.ectively (Fig. 11B and Table. 5). Similar results were obtained for the melanin obtained from Catharsius molossus [79].

Table 5. Differential Scanning calorimetric analysis for extracellular melanin from Prauserellasp.. (MAPPL 017A) 


\begin{tabular}{|c|c|c|c|}
\hline & Start and end temperature & Maximum & Area \\
\hline S. No. & Degree Celsius & Degree Celsius & $\mathrm{J} / \mathrm{g}$ \\
\hline 1. & $85.04-111.22$ & 91.47 & 0.06059 \\
\hline 2. & $210.61-247.57$ & 229.61 & 86.44 \\
\hline 3. & $272.79-309.80$ & 282.65 & 23.62 \\
\hline
\end{tabular}

\section{Conclusion}

Melanin the natural biopolymer which plays an important role in most living organisms. Based on the physical and chemical composition it has been isolated and purified from different sources and the exact structure of the melanin was not yet identified. Among the different types of melanin, eumelanin is the predominant one. Sepia melanin is well-known eumelanin purified from cuttlefish (Sepia officinalis) which was completely characterized by many of the researchers and it occupies a foremost position in melanin industries. The second position was occupied by fungi followed by bacterial melanin but only a few reports are available on actinomycetes. In the present study, the melanin pigment purified and characterized from the novel actinomycetes Prauserella sp. and it was confirmed as eumelanin based on the color and chemical nature. For the additional support, the enhanced production of melanin by the isolate Prauserella sp. was observed only in the presence of tyrosine through DOPA pathway thus confirmed that the resultant eumelanin was DOPA type. The various biological activities of the melanin from Prauserella sp. can be used various fields like cosmetics, bioelectronics, semiconductors, anticancer drugs, and protective equipment.

\section{Declarations}

\section{Conflict of Interest}

We know of no conflicts of interest with this publication and there has been no significant finance support for this work that could have influenced its outcome.

\section{Acknowledgement}

The author would like to acknowledge the instrument facilities provided by SAIF-IITM, NMR centers IISC Bangalore and Caters CLRI Chennai, and Instrument facilities at University of Madras.

\section{References}

1. Keisham Singh, Mahesh S. MajikSupriya Tilvi,Vibrational Sp.ectroscopy for Structural Characterization of Bioactive Compounds (Chapter 6).Comprehensive Analytical Chemistry 65 (2014), 115-148. DOI: 10.1016/B978-0-444-63359-0.00006-9. 
2. K. Tarangini, S. Mishra, Production, characterization and analysis of melanin from isolated marine Pseudomonas sp.. using vegetable waste, Research Journal of Engineering Sciences, 2(5) (2013) 4046.

3. K.M. Langfelder, B. Streibel, G. Jahn, A.A. Haase, Brakhage, Melanins, Annu. Rev. Phytopath. 24 (2003) 411-451.

4. P.A. Riley, Melanogenesis: A realistic target for antimelanoma therapy Eu. J, Can. 27(1991) 11711174.

5. K. Ikeda, K. Suzuki, H. Yoshioki, K. Miyamoto, T. Masujima, M Sugiyama, Construction of a new cloning vector utilizing a cryptic plasmid and the highly expressed melanin-synthesizing gene operon from Streptomyces castaneoglobisp.orus. FEMS Microbiol Lett 168 (1998) 195-199.

6. Pragat Agarwal, Muta Singh, Jyoti Singh, R.P. Singh, Microbial tyrosinase: A novel enzyme structural fetures and applications (Chapter 1). Applied Microbiology and bioengineering an interdisciplinary approach, (2019), 3-19.

7. M.P. Przemyslaw, G. Maja, Melanin synthesis in microorganisms: Biotechnological and medical asp.ects, Acta. Biochem. Pol. 53 (2006) 429-443.

8. R. Romero-Martinez, M. Wheeler, A. Guerrero-Plata, G. Rico, H. Torres-Guerrero, Biosynthesis and functions of melanin in Sp.orothrix schenckii, Infect Immun. 68 (2000) 3696-3703.

9. R. Zanetti, G. Prota, A. Napolitano, C. Martinez, H. Sancho-Garnier, A. Osterlind, C. Sacerdote, S. Rosso, Development of an integrated method of skin phenotype measurement using the melanins, Melanoma Res. 11 (2001) 551-557.

10. E.S. Jacobson, Pathogenic roles for fungal melanins, Clin. Microbiol. Rev. 13 (2000) 708-717.

11. Y. Mikarni. K. Yokoyama, T. Arai, Modified Arai and Mikami melanin formation test of Streptomycetes, International Journal of Systematic Bacteriology, 1977, p. 290

12. S. Palm, L.F. Linhares, A.S. Mangrich, J.P. Martin, Characterization of fungal melanins and soil humic acids by chemical analysis and infrared sp.ectroscopy, Biol. Fertil. Soils, 10 (1990) 72-76.

13. F. Fava, D.D. Gioia, L. Merchetti, Characterization of a pigment produced by Pseudomonas fluorescens during 3-chlorobenzoate co-metabolism. Chemosp.here, 27 (1993) 825-835.

14. H. Wang, Y. Pan, X. Tang, Z. Huang, Isolation and characterization of melanin from Osmanthus fragrans seeds, LWT-Food Science and Technology, 39 (2006) 496-502.

15. W.T. Dixon. Sp.inning-sideband-free and sp.inning-sideband-only NMR sp.ectra in sp.inning samples, J. Chem. Phys. 77 (1982) 1800.

16. B.M. Fung, A.K. Khitrin, K. Ermolaev, An improved broadband decoupling sequence for liquid crystals and solids, J. Magn. Reson. 142(1) (2000) 97-101.

17. M.S. Blois, H.W. Brown, J.E. Maling, Free Radicals in Biological Systems, (M.S. Blois, Jr., H.W. Brown, R.M. Lemmon, and M. Weissbluth, editors), New York, 1961. Academic Press, Inc., 117.

18. V.M. Sava, S.M.Yang, M.Y. Hong, P.C. Yang, G.S. Huang, Isolation and characterization of melanic pigments derived from tea and tea polyphenols, Food Chem. 73(2) (2001b) 177-184. 
19. J. Geng, W. Tang, X. Wan, Q. Zhou, X.J. Wang, P. Shen, T.C. Lei, X.D. Chen, Photoprotection of bacterial-derived melanin against ultraviolet A-induced cell death and its potential application as an active sunscreen, European Academy of Dermatology and Venereology, 22 (2008) 852-858.

20. J. Schmaler-Ripcke, V. Sugareva, P. Gebhardt, R. Winkler, O. Kniemeyer, T. Heinekamp, A.A. Brakhage, Production of pyomelanin, a second type of melanin, via the tyrosine degradation pathway in Asp.ergillus fumigatus, App. Environ. Microbiol. 75(2) (2009) 493-503.

21. S.S. Sajjan, G.B.Kulkarni, V. Yaligara, L. Kyoung, T.B. Karegoudar, Purification and physiochemical characterization of melanin pigment from Klebsiella sp.. GSK, J Microbiol. Biotechnol. 20 (2010) 1513-1520.

22. R.C.R. Goncalves, H.C.F. Lisboa, S.R. Pombeiro-Sp.onchiado, Characterization of melanin pigment produced by Asp.ergillus nidulans, World J Microbiol Biotechnol. 28 (2012) 1467-1474.

23. Ming Ye, Yan Wang, Meishuang Qian, Xiao Chen, Xueqin Hu, Preparation and Properties of the Melanin from Lachnum singerianum, International Journal of Basic and Applied Sciences, 11(3) (2011) 51-58.

24. C. Ganesh Kumar, P. Mongolla, S. Pombala, A. Kamle, J. Joseph, Physicochemical characterization and antioxidant activity of melanin from a novel strain of Asp.ergillus bridgeri ICTF-201, Letters in Applied Microbiology, 53 (2011) 350-358.

25. R. Almeida-Paes, S. Frases, S., Araujo Gde, M.M. de Oliveira, G.J. Gerfen, J.D. Nosanchuk, R.M. Zancope-Oliveira,. Biosynthesis and functions of a melanoid pigment produced by sp.ecies of the Sp.orothrix complex in the presence of L-tyrosine, Appl Environ Microbiol. 78 (2012) 8623-8630.

26. Caihong Dong, Yijian Yao, Isolation, characterization of melanin derived from Ophiocordyceps sinensis, an entomogenous fungus endemic to the Tibetan Plateau, Journal of Bioscience and Bioengineering, 113(4) (2012) 474-479.

27. Mugdha Apte, Gauri Girme, Ashok Bankar, Ameeta Ravikumar, Smita Zinjarde, 3,4-dihydroxy-Lphenylalanine-derived melanin from Yarrowia lipolytica mediates the synthesis of silver and gold nanostructures, Journal of Nanobiotechnology, 11(2) (2013) 3-11.

28. C. Ganesh Kumar, N. Sahu, G. Narender Reddy, R.B.N. Prasad, N. Nagesh, A. Kamal Production of melanin pigment from Pseudomonas stutzeri isolated from red seaweed Hypnea musciformis, Letters in Applied Microbiology, 57 (2013) 295-302.

29. Mohamed Tawfiek Shaaban, Sabha Mahmoud Mabrouk El-Sabbagh, Asmaa Alam, Studies on an Actinomycete producing a melanin pigment inhibiting Aflatoxin B1 Production by Asp.ergillus flavus, Life Sci. J. 10(1) (2013) 1437-1448.

30. Jing Guo, Zhiming Rao, Taowei Yang, Zaiwei Man, Meijuan Xu, Xian Zhang, High-level production of melanin by a novel isolate of Streptomyces kathirae, FEMS Microbiol Lett. 357 (2014) 85-91.

31. Ming Ye, Geng-yi Guo, Ying Lu, Sheng Song, Hui-yan Wang, Liu Yang, Purification, structure and antiradiation activity of melanin from Lachnum YM404, International Journal of Biological Macromolecules, 63 (2014) 170-176. 
32. George Seghal Kiran, Asha Dhasayan, Anuj Nishanth Lipton, Joseph Selvin, Mariadhas Valan Arasu, Naif Abdullah Al-Dhabi, Melanin-templated rapid synthesis of silver nanostructures, Journal of Nanobiotechnology, 12(18) (2014) 2-13.

33. G. Arun M. Eyini, P. Gunasekaran, Characterization and biological activities of extracellular melanin produced by Schizophyllum commune (Fries), Indian Journal of Experimental Biology, 53 (2015) 380387.

34. Sun Xiujun, Wu Biao, Zhou Liqing, Liu Zhihong, Dong Yinghui, Yang Aiguo, Isolation and Characterization of melanin pigment from Yesso Scallop Patinopecten yessoensis. Ocean Univ. China (Oceanic and Coastal Sea Research), 16(2) (2017) 279-284.

35. J. Krysciak, Solubility of melanin and humin preparations in polar aromatic solvents, Folia. Biol. 33 (1985) 195-214.

36. A.A. Bell, M.H. Wheeler, Biosynthesis and functions of fungal melanins, Annual Review of Phytopathology, 24 (1986) 411-451.

37. F.G. Lewis, M.G. Antony, Melanin and novel-melanin precursors from Aeromonas media, FEMS Microbiol. Lett. 169 (1998) 261-268.

38. E.C. Vernon, A. Lena, Induction of melanin biosynthesis in Vibrio cholera, Appl. Environ. Microbiol. 58(9) (1992) 2861-2865.

39. A. Gibello, M. Suárez, A.J. Luis, M. Martín, Molecular cloning and analysis of the genes encoding the 4-hydroxyphenylacetate hydroxylase from Klebsiella pneumoniae. Arch. Microbiol., 167 (1997) 160166.

40. V.M. Sava, B.N. Galkin, M.Y. Hong, P.C. Yang, G.S. Huang, A novel melanin- like pigment derived from black tea leaves with immuno-stimulating activity, Food Research International. 34(4) (2001a) 337343.

41. V.H.N. Lagunas-Munoz, N. Cabrera-Valladares, F. Bolivar, G. Gosset, A. Martinez, Optimum melanin production using recombinant Escherichia coli, J. Appl. Microbiol. 101 (2006) 1002-1008.

42. N. Christine, S. Santos S. Gregory, Melanin-based -high-throughput screen for L-tyrosine production in Escherichia coli, Appl. Environ. Microbiol. 74 (2008) 1190-1197.

43. Y. Huang, X. Lai, X. He, L. Cao, Z. Zeng, J. Zhang, S. Zhou, Characterization of a deep-sea sediment metagenomic-clone that produces water-soluble melanin in Escherichia coli. Mar. Biotechnol. 11 (2009) 124-131.

44. Y.H.B. Wenlin Stephen O.D. Jeffrey, Melanin biosynthesis by Frankia strain Cel5, Physiol. Plant. 131 (2007) 180-190.

45. P. Selvakumar, S. Rajasekar, K. Periasamy, N. Raaman, Isolation and characterization of melanin pigment from Pleurotus cystidios (telomorph of Antromyocopsis macrocapa), World J. Microbiol. Biotechnol. 24 (2008) 2125-2131.

46. Y. Tu, Y. Sun, Y. Tian, M. Xie, J. Chen, Physicochemical characterization and antioxidant activity of melanin from the muscles of Taihe Black-bone silky fowl (Gallus gallus domesticus Brisson), Food Chemistry, 114 (2009) 1345-1350. 
47. P. Meredith, B.J. Powell, J. Riesz, S. Nighswander-Rempel, M.R. Pederson, E. Moore, Towards structure property function relationships for eumelanin, Soft Matter. 2 (2006) 37-44.

48. M.L. Wolbarsht, A.W.Wash, G. George, Melanin, a unique biological absorber, Appl. Opt. 20 (1981) 2184-2186.

49. H.C. Longuet-Higgins, On the origin of the free radical properties of melanin, Arch Biochim. Biophys. Acta. 186 (1960) 231-232.

50. J. Lindon, G. Tranton, J. Holmes, Bio-macromolecular application of UV-Vis absorption sp.ectroscopy Elsevier, (2000) pp.131-136.

51. P.R. Crippa, V. Cristofoletti, N. Romeo, A band model for melanin deduced from optical absorption and photoconductivity measurements, Biochim. Biophys. Acta. 538 (1978) 164-170.

52. M.L. Tran, B.J. Powell, P. Meredith, Chemical and structural disorder in eumelanins: a possible explanation for broadband absorbance, Biophys. J. 90 (2006) 743-752.

53. T.G. Booner, A. Duncan, Infra-red sp.ectra of some melanins, Nature, 194 (1962) 1078-1079.

54. A.R. Katritzky, N.G. Akhmedov, S.N. Denisenko, O.V. Denisko, ${ }^{1}$ H NMR sp.ectroscopic characterization of solutions of Sepia melanin, Sepia melanin free acid and human hair melanin, Pigment Cell Research, 15(2) (2002) 93-97.

55. S.A. Centeno, J. Shamir, Surface enhanced Raman scattering (SERS) and FTIR characterization of the sepia melanin pigment used in works of art, Journal of Molecular Structure, 873 (2008) 149-159.

56. F. Bernsmann, O. Ersen, J.C. Voegel, N. Jan, A. Kotov, V. Ball, Melanin-containing films: Growth from dopamine solutions versus layer-by-layer deposition, Chem Phys Chem. 11 (2010) 3299-3305.

57. A. Mbonyiryivuze, Z.Y. Nuru, L. Kotsedi, B. Mwakikunga, S.M. Dhlamini, E. Park, M. Maaza, Multi-scale assembly in nano-scaled sepia melanin natural dye. Materials Today: Proceedings, 2 (2015) 39883997.

58. J.-W. Lee, H.-B. Cho, T. Nakayama, T. Sekino, S.-I. Takana, K. Minato, T. Ueno, T. Suzuki, H. Suematu, T. Yoshinori and K. Niihara, "Dye-sensitized solar cells using purified squid ink nanoparticles coated on TiO2 nanotubes/nanoparticles, Journal ofceramic Society of Japan, 121(1), (2013) 123-127.

59. J. Manivasagan, K. Venkatesan, K. Senthilkumar, K. Sivakumar, S.-K. Kim, Isolation and characterization of biologically active melanin from Actinoalloteichus sp.. MA-32, International Journal of Biological Macromolecules, 58, (2013) 263-274.

60. Shuai Zong, Lan Li, Jinglei Li, Farnaz Shaikh, Li, Yang, Ming Ye, Structure Characterization and Lead Detoxification Effect of Carboxymethylated Melanin Derived from Lachnum sp., Appl. Biochem. Biotechnol. 182(2) (2017) 669-686.

61. MG. Bridelli, D. Tampellini, L. Zecca, The structure of neuromelanin and its iron binding site studied by infrared sp.ectroscopy. FEBS Lett. 457 (1999) 18-22.

62. A.M. Gomez-Marin, C.I. Sanchez, Thermal and mass sp.ectroscopic characterization of a sulphurcontaining bacterial melanin from Bacillus subtilis. Journal of Non-Crystalline Solids, 365 (31-32) (2010) 1576-1580. 
63. M. Magarelli, Purification, characterization and photodegradation studies of modified sepia melanin Sepia (Sepia officinalis), Determination of Eumelanin content in fibers from Alpaca (Vicugna pacos), Doctoral Thesis., 2011, Macerata: University of Camerino.

64. L. Bardani, M.G. Bridelli, M. Carbucicchio, P.R. Crippa, Comparative mossbauer and infrared-analysis of iron containing melanins, Biochim Biophys Acta. 716 (1982) 8-15.

65. L. Hong, D. Simon, Insight into the binding of divalent cations to sepia eumelanin from IR absorption sp.ectroscopy, Photochem. Photobiol. 82(5) (2006) 1265-1269.

66. M. Magarelli, P. Passamonti, C. Renieri, Purification, characterization and analysis of sepia melanin from commercial sepia ink (Sepia Officinalis), CES Medicina Veterinaria y Zootecnia, 5(2) (2010) 1828.

67. S. Matsunuma, Theoretical simulation of resonance Raman bands of amorphous carbon, Thin Solid Films 306 (1997) 17.

68. V. Capozzi, G. Perna, A. Gallone, P.F. Biagi, P. Carmone, A. Fratello, G. Guida, P. Zanna, R. Cicera, Raman and optical sp.ectroscopy of eumelanin films, J. Mol. Struct. 744 (2005) 717.

69. A. Nagano, T. Yoshitake, T. Hara, K. Nagayama, Optical properties of ultrananocrystalline diamond/amorphous carbon composite films prepared by pulsed laser deposition, Diamond Relat. Mater. 17 (2008) 1199.

70. Priti Vairale, Vaishali Waman, Azam Mayabadi, Habib Pathan, Sandesh Jadkar, Electrochemical Synthesis of Melanin Thin Films: Evolution of Structural and Optical Properties, International Journal of Advanced Research in Physical Science (IJARPS) 1(7) (2014) 35-45.

71. Zhiwei Huang, Harvey Lui, Chen Abdulmajeed Alajlan X.K., David I. McLean, Haishan Zeng, Raman sp.ectroscopy of in vivo cutaneous melanin. Journal of Biomedical Optics 9(6), (2004) 1198-1205.

72. A.L. Rosas, J.D. Nosanchuk, B.L. Gómez, W.A. Edens, J.M. Henson, A. Casadevall, Isolation and serological analyses of fungal melanins, Journal of Immunological Methods, 244 (2000) 69-80.

73. B.L. Gomez, J.D. Nosanchuk, S. Díez, S. Youngchim, P. Aisen, L.E. Cano, A. Restrepo, A. Casadevall, A.J. Hamilton, Detection of melanin-like pigments in the dimorphic fungal pathogen Paracoccidioides brasiliensis in vitro and during infection, Infect Immun., 69 (2001) 5760-5767.

74. J.D. Nosanchuk, B.L. Gomez, S. Youngchim, S. Diez, P. Aisen, R.M. Zancope-Oliveira, A. Restrepo, A. Casadevall, A.J. Hamilton, Histoplasma capsulatum synthesizes melanin-like pigments in vitro and during mammalian infection, Infect Immun. 70 (2002) 5124-5131.

75. R. Morris-Jones, S. Youngchim, B.L. Gomez, P. Aisen, R.J. Hay, J.D. Nosanchuk, A. Casadevall, A.J. Hamilton, Synthesis of melanin-like pigments by Sp.orothrix schenckii in vitro and during mammalian infection, Infect. Immun. 71 (2003) 4026-4033.

76. Toshihiko Matsuura, Midori Hino, Sei-Ichi Akutagawa, Yuhei Shimoyama, Takanori Kobayashi, Yoshihiro Taya,Takashi UENO, Optical and Paramagnetic Properties of Size-controlled ink particles isolated from Sepia officinalis, Bioscience Biotechnology and Biochemistry, 73(12) (2009) 27902792. 
77. W.S. Enochs, M.J. Nilges, H.M. Swartz, A standardized test for the identification and characterization of melanin using electron paramagnetic resonance (EPR) sp.ectroscopy, Pigment Cell Res. 6 (1993) 91-99.

78. P. Jalmi, P. Bodke, S. Wahidullah, S. Raghukumar, The fungus Gliocephalotrichum simplex as a source of abundant, extracellular melanin for biotechnological applications, World Journal of Microbiology and Biotechnology, 28(2) (2012) 505-512.

79. Chao Xin, Jia-hua Ma, Cheng-jia Tan, Zhou Yang, Feng Ye, Chan Long, Shuang Ye, and Da-bin Hou, Preparation of melanin from Catharsius molossus L. and preliminary study on its chemical structure, Journal of Bioscience and Bioengineering, 119(4) (2014) 1-9.

80. E.S. Bronze-Uhle, A. Batagin-Neto, P.H.P. Xavier, N.I. Fernandes, E.R. de Azevedo, C.F.O. Graeff, Synthesis and characterization of melanin in DMSO, J. Mol. Struct. 1047 (2013) 102-108.

81. B.B. Adhyaru, N.G. Akhmedov, A.R. Katritzky, C.R. Bowers, Solid-state cross-polarization magic angle sp.inning ${ }^{13} \mathrm{C}$ and ${ }^{15} \mathrm{~N}$ NMR characterization of Sepia melanin, Sepia melanin free acid and human hair melanin in comparison with several model compounds, Magn. Reson. Chem. 41 (2003) 466-474.

82. Rafael Prados-Rosales, Stacy Toriola, Antonio Nakouzi, Subhasish Chatterjee, Ruth Stark, Gary Gerfen, Paul Tumpowsky, Ekaterina Dadachova, and Arturo Casadevall Structural Characterization of Melanin Pigments from Commercial Preparations of the Edible Mushroom Auricularia auricular, J. Agric. Food Chem. 63 (2015) 7326-7332.

83. S. Aime, B. Bergamasco, M. Casu, G. Digilio, M. Fasano, S. Giraudo, L. Lopiano, Isolation and ${ }^{13} \mathrm{C}-$ NMR Characterization of an Insoluble Proteinaceous Fraction from Substantia Nigra of Patients with Parkinson's Disease, Movement Disorders, 15(5) (2000) 977-981.

84. S. Chatterjee, R. Prados-Rosales, S. Frases, B. Itin, A. Casadevall, R.E. Stark, Using solid-state NMR to monitor the molecular consequences of Cryptococcus neoformans melanization with different catecholamine precursors, Biochemistry, 51 (2012) 6080-8.

85. S. Chatterjee, R. Prados-Rosales, S. Tan, B. Itin, A. Casadevall, R.E. Stark, Demonstration of a common indole-based aromatic core in natural and synthetic eumelanins by solid-state NMR, Org. Biomol. Chem. 12 (2014) 6730-6.

86. G.A. Duff, J.E. Roberts, N. Foster, Analysis of the structure of synthetic and natural melanins by solidphase NMR. Biochemistry, 27 (1988) 7112-7116.

87. M.G. Peter H. Forster, On the structure of eumelanins: Identification of constitutional patterns by solid-state NMR sp.ectroscopy, Angewandte Chemie, International Edition, English, 28(6) (1989) 741.

88. S. Aime, M. Fasano, C. Croombridge, Solid-state ${ }^{13} \mathrm{C}$ NMR characterization of melanin free acids from biosynthetic and natural melanins, Gazzetta Chimica Italiana 120 (1990) pp. 663.

89. Keely Glass, Shosuke Ito, Philip R. Wilby, Takayuki Sota, Atsushi Nakamura, C. Russell Bowers, Jakob Vinther, Suryendu Dutta, Roger Summons, Derek E. G. Briggs, Kazumasa Wakamatsu, John D. Simon, Direct chemical evidence for eumelanin pigment from the Jurassic period, PNAS, 109 (2012) 26. 
90. D.N. Moses, J.H. Harreld, G.D. Stucky, J.H. Waite, Melanin and Glycera jaws emerging dark side of a robust biocomposite structure, J. Biol. Chem. 281 (2006) 34826-34832.

91. S. Ito, K. Fujita, Microanalysis of eumelanin and pheomelanin in hair and melanomas by chemical degradation and liquid chromatography, Anal. Bio- chem. 144 (1985) 527-536.

92. K. Wakamatsu, S. Ito, Advanced chemical methods in melanin determination, Pigment Cell Res. 15 (2002) 174-183.

93. M. Piattelli, E. Fattorusso, S. Magno, R.A. Nicolaus, Ustilago melanin, a naturally occurring catechol melanin, Tetrahedron Lett. 4(15) (1963) 997-998.

94. H. Stussi, D.M. Rast, The biosynthesis and possible functions of $\mathrm{y}$-glutaminyl-4-hydroxybenzene in Agaricus bisp.orus, Phytochemistry, 20 (1981) 2347-2352.

95. S. Ito, K. Wakamatsu, Chemical degradation of melanins: application to dopamine-melanin, Pigment Cell Res. 11 (1998) 120-126.

96. C.S. Alviano, S.R. Farbiarz, W. De Souza, J. Angluster, L.R. Travassos, Characterization of Fonsecaea pedrosoi melanin, J. Gen. Microbiol. 137 (1991) 837-844.

97. Y. Wang, P. Aisen, A. Casadevall, Melanin, melanin 'ghosts', and melanin composition in Cryptococcus neoformans, Infect. Immun. 64 (1996) 2420-2424.

98. E. Dadachova, R.A. Bryan, X. Huang, T. Moadel, A.D. Schweitzer, P. Aisen, J.D. Nosanchuk, A. Casadevall, lonizing radiation changes the electronic properties of melanin and enhances the growth of melanized fungi, PLOS ONE, 5 (2007) 457.

99. Si-Rui Chen, Bin Jiang, Jiang-Xia Zheng, Gui-Yun Xu, Jun-Yin Li, Ning Yang, Isolation and characterization of natural melanin derived from silky fowl (Gallus gallus domesticus Brisson), Food Chemistry, 111 (2008) 745-749.

100. V. Capozzi, G. Perna, P. Carmone, A. Gallone, M. Lastella, E. Mezzenga, G. Quartucci, M. Ambrico, V. Augelli, P.F. Biagi, T. Ligonzo, A. Minafra, L. Schiavulli, M. Pallara, R. Cicero, Optical and photoelectronic properties of melanin, Thin Solid Films, 511 (2006) 362-366.

101. S. Reale, M. Crucianelli, A. Pezzella, M. d'Ischia, F. De Angelis, Exploring the frontiers of synthetic eumelanin polymers by high-resolution matrix-assisted laser/desorption ionization mass sp.ectrometry, J. Mass. Sp.ectrom. 47(1) (2012) 49-53.

102. Monika Varga, Otto Berkesi, Zsuzsanna Darula, Nora Veronika May, Andras Palagyi, Structural characterization of allomelanin from black oat. Phytochemistry, 130 (2016) 313-320.

103. A.H. Soeriyadi, M.R. Whittaker, C. Boyer, T.P. Davis, Soft-lonization Mass Sp.ectroscopy: Insights into the Polymerization Mechanism, J. Polym. Sci. Part A, 51 (2013) 1475-1505.

104. C. Wesdemiotis, Multidimensional Mass Sp.ectrometry of synthetic polymers and advanced materials, Angew. Chem. Int. Ed., 129 (2017) 1474-1487.

\section{Figures}





\section{Figure 1}

Colony morphology and production of melanin in/on liquid and solid medium of Czapek tyrosine. A: colony morphology of MAPPL 017A; B: Production of melanin in Czapek tyrosine broth; C\&D: Aerial (C) and substrate (D) view of melanin production on Czapek tyrosine agar. 


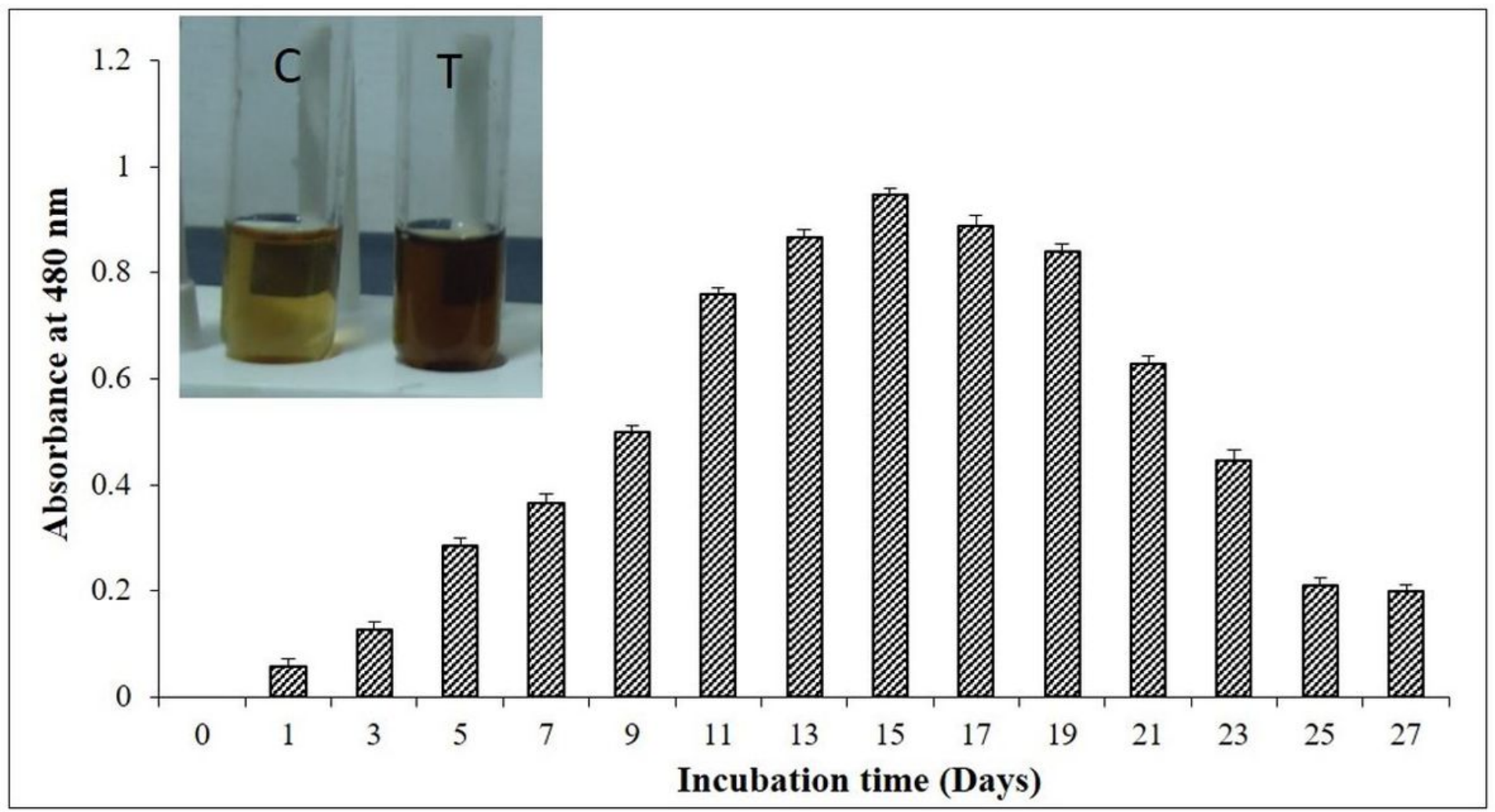

Figure 2

Qualitative and quantitative determination of tyrosinase activity by Prauserella sp. at different time intervals 

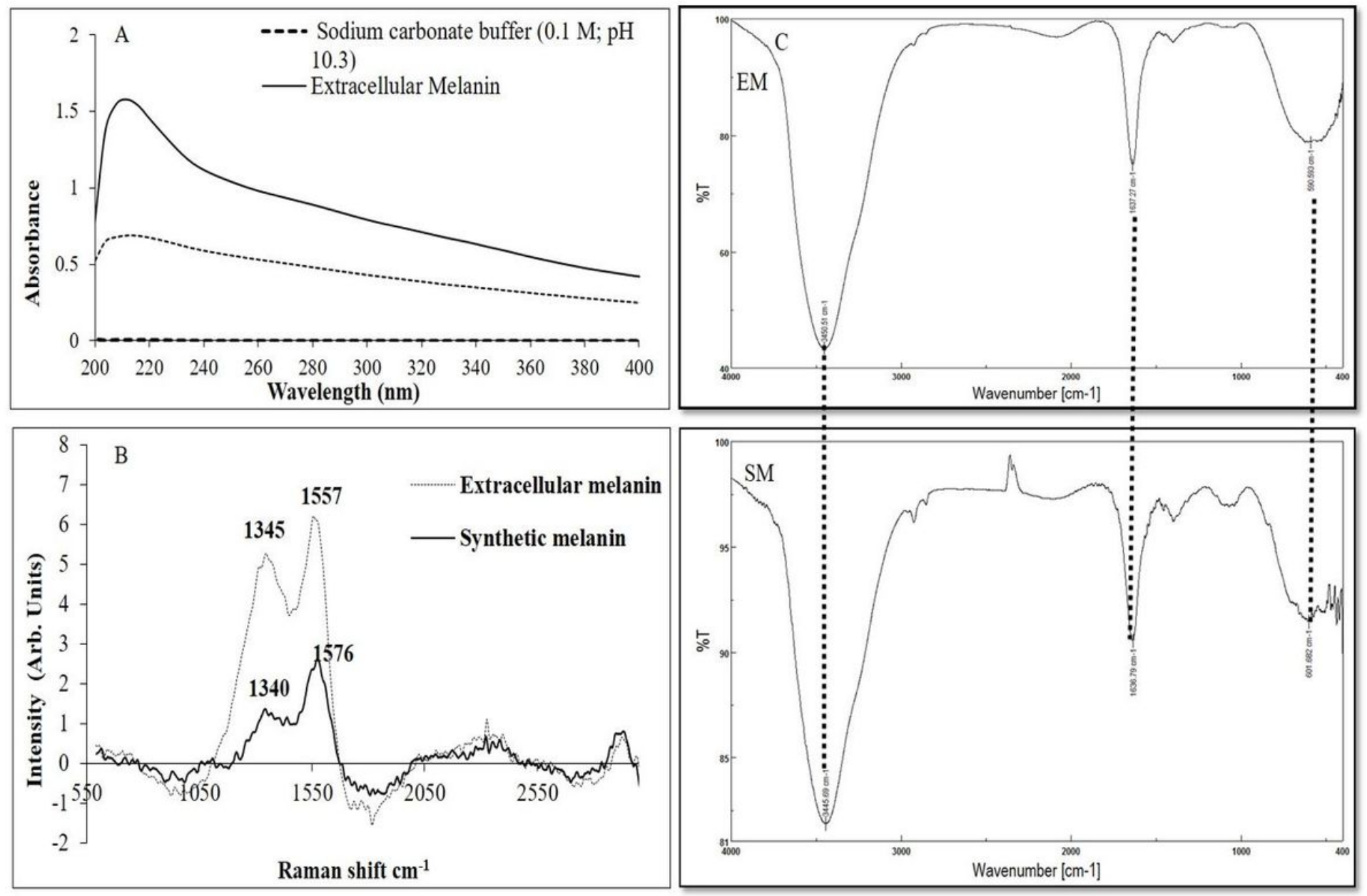

Figure 3

Sp.ectroscopic analysis of extracellular melanin from Prauserella sp. A: UV-VIS sp.ectroscopic analysis; B: Raman Sp.ectroscopic analysis; C: FTIR Sp.ectral analysis of purified and Synthetic melanin. 

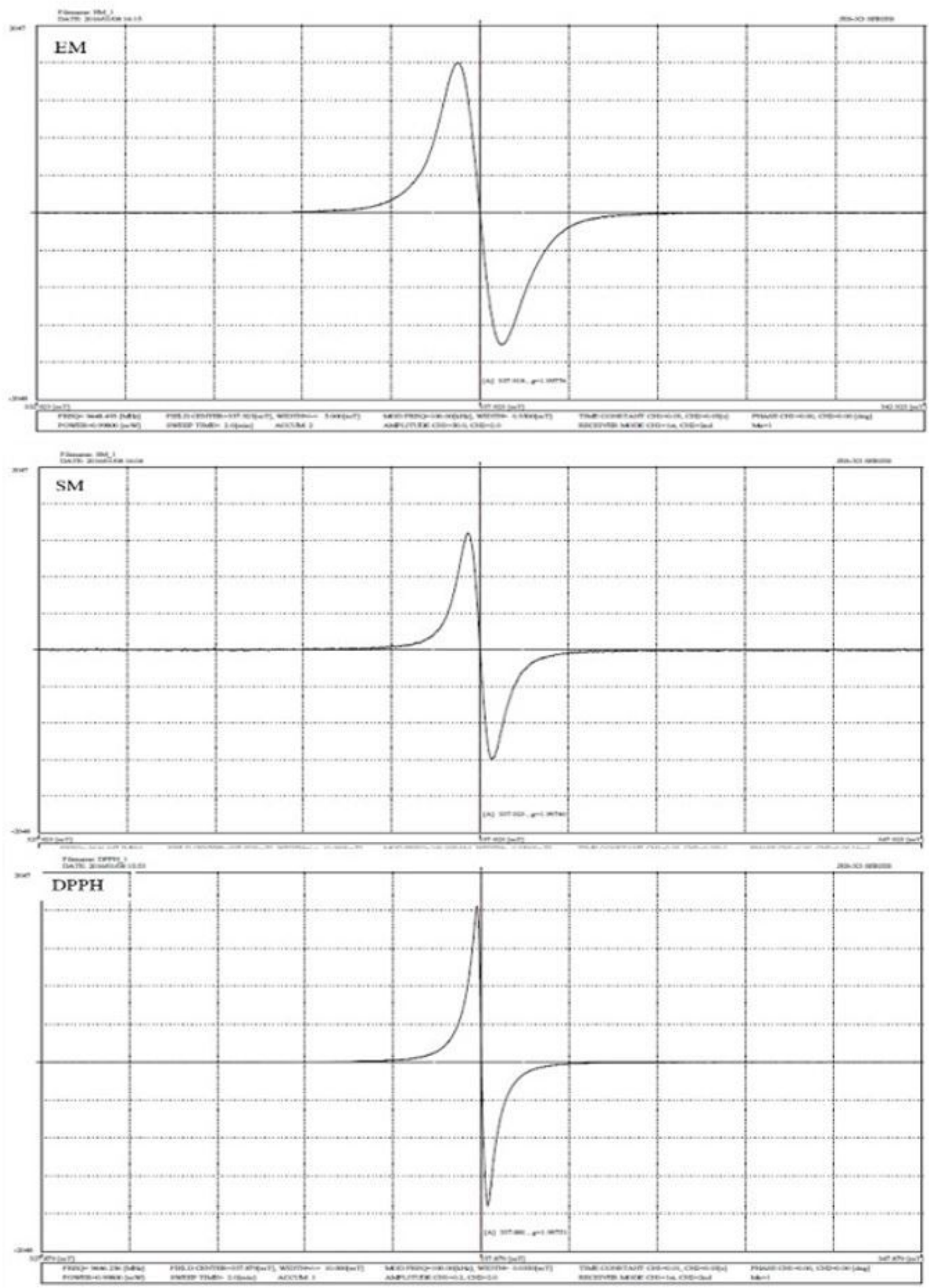

\section{Figure 4}

Electro paramagnetic resonance sp.ectroscopic analysis of extracellular melanin of Prauserella sp. (MAPPL 017A) 

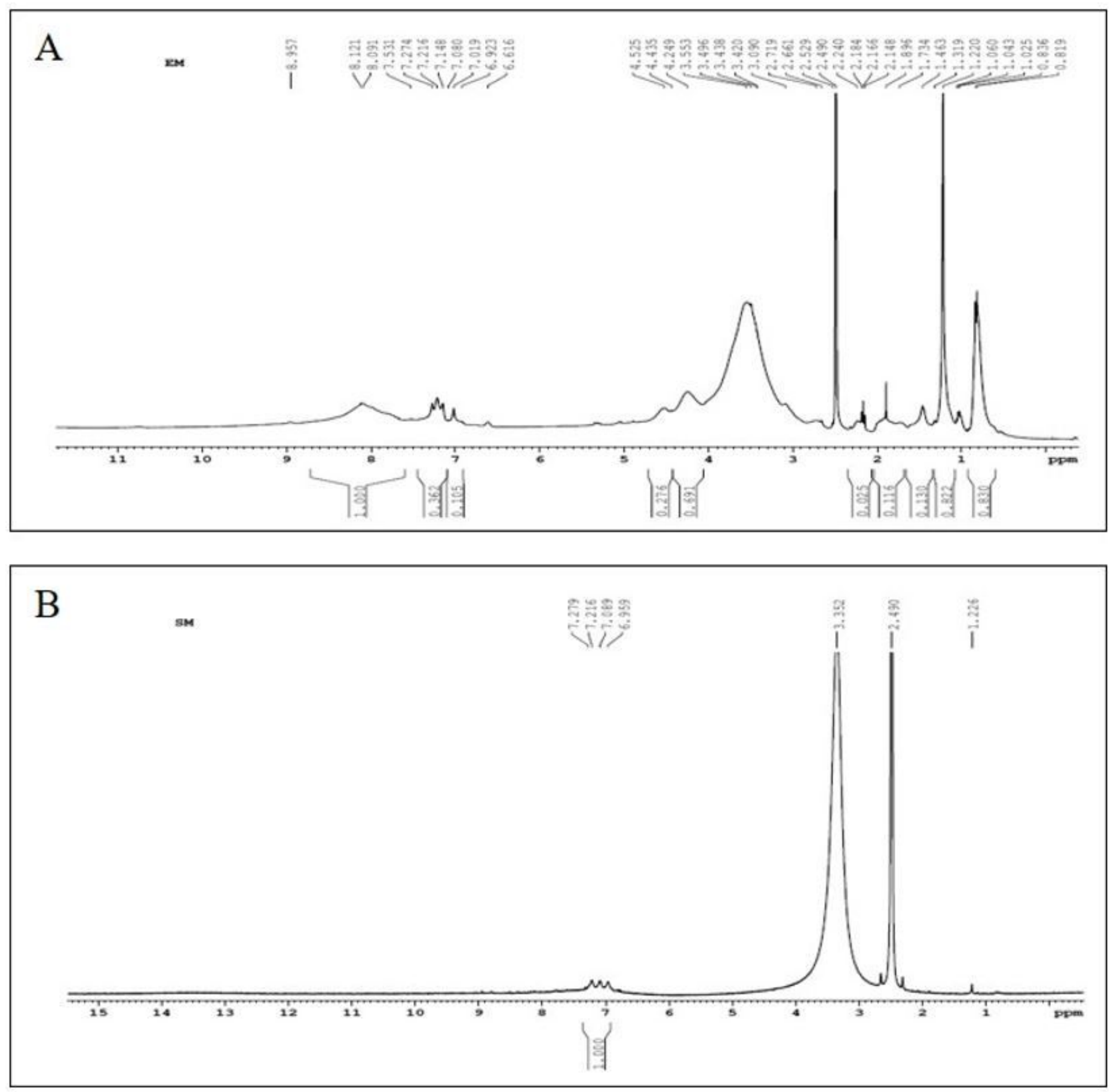

Figure 5

1H NMR sp.ectral analysis of extracellular melanin from Prauserella sp.. (MAPPL 017A) and Synthetic melanin using D2O/NH4OH at pH 10.0 A: Extracellular melanin from Prauserella sp..; B: Synthetic melanin 


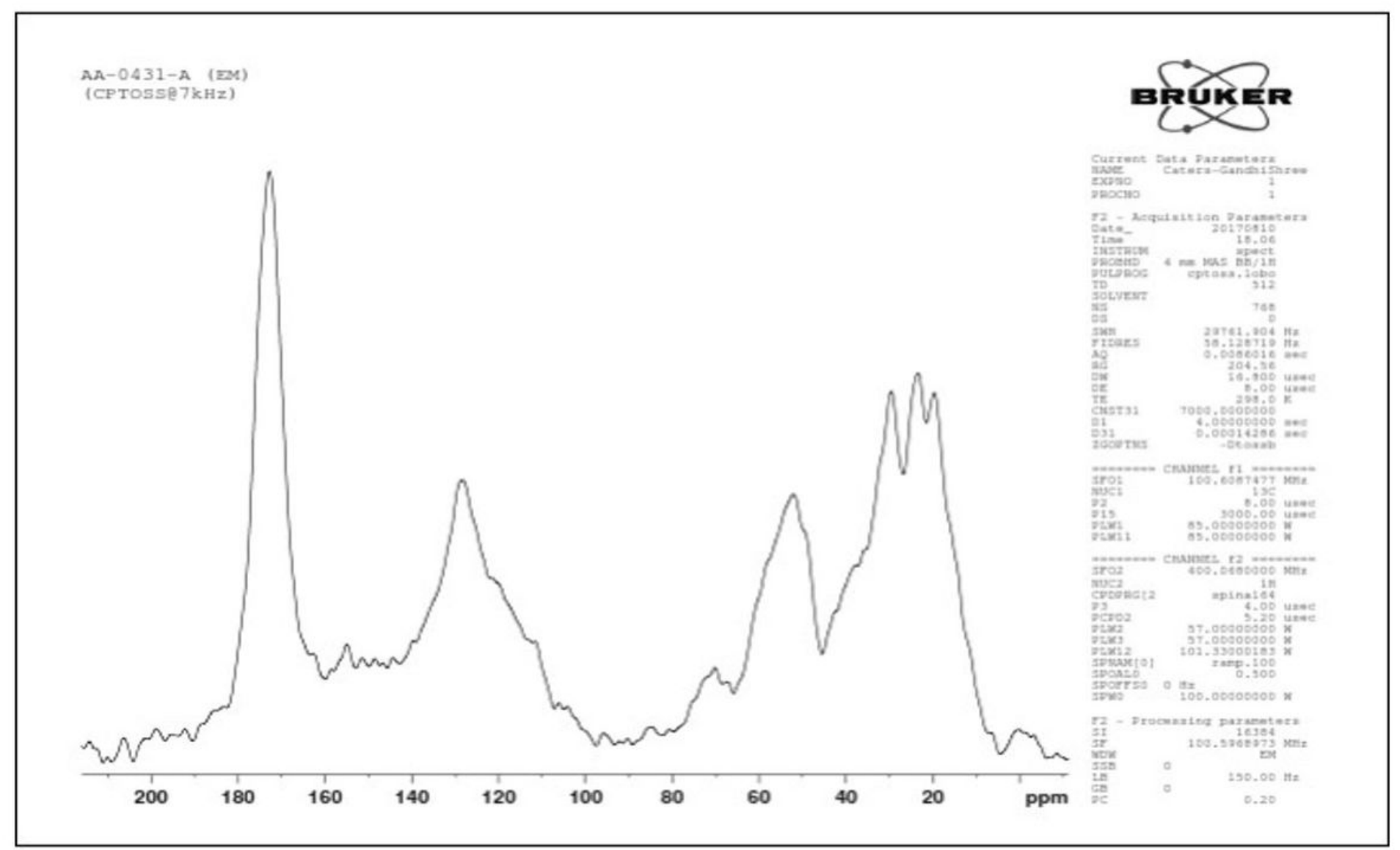

Figure 6

13C CP/MAS sp.ectral analysis of extracellular melanin from Prauserella sp.. (MAPPL 017A) 

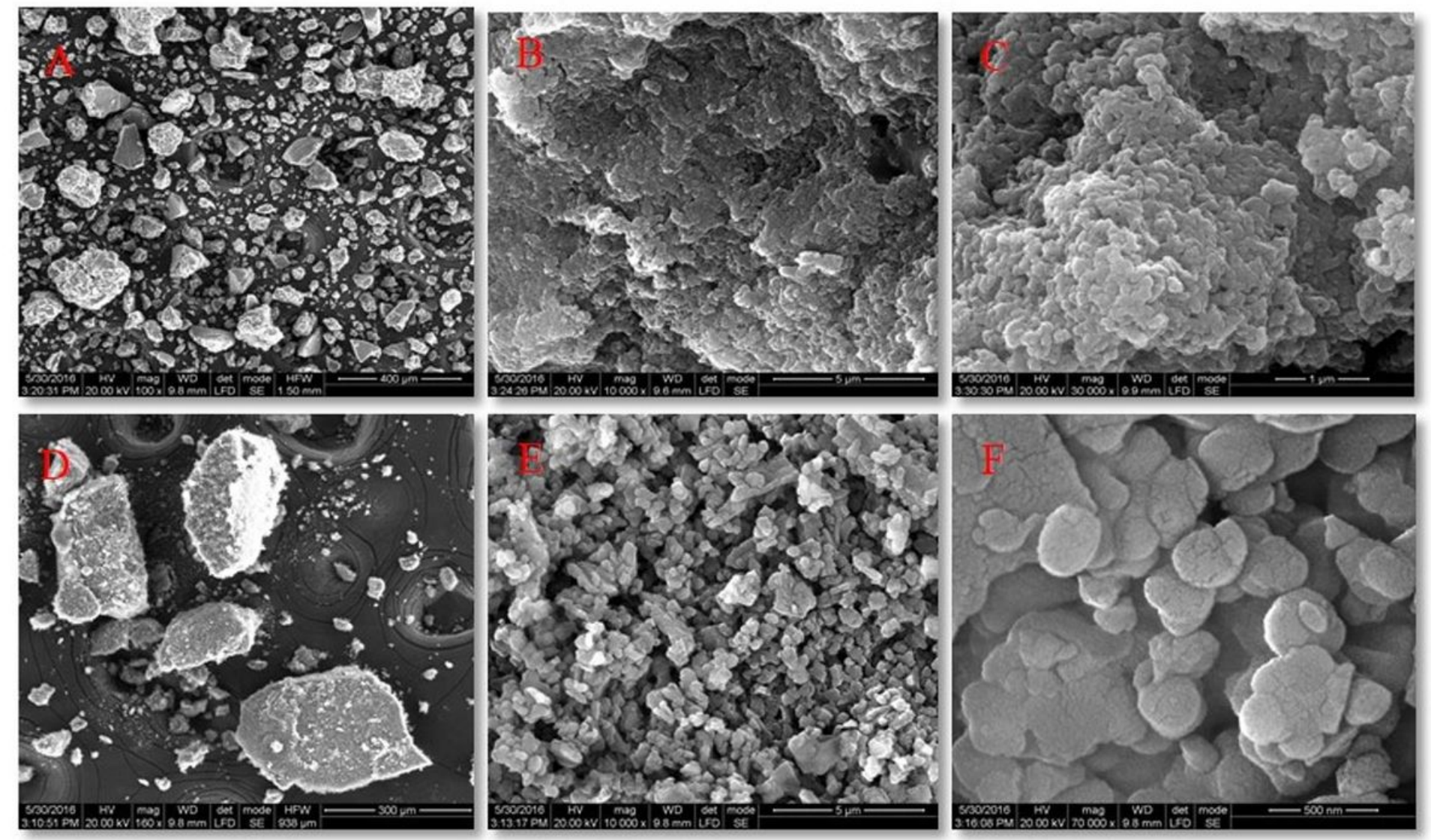

Figure 7

SEM micrographs of extracellular melanin (A-C) from Prauserella sp.. (MAPPL 017A) and Synthetic melanin (D-F) 

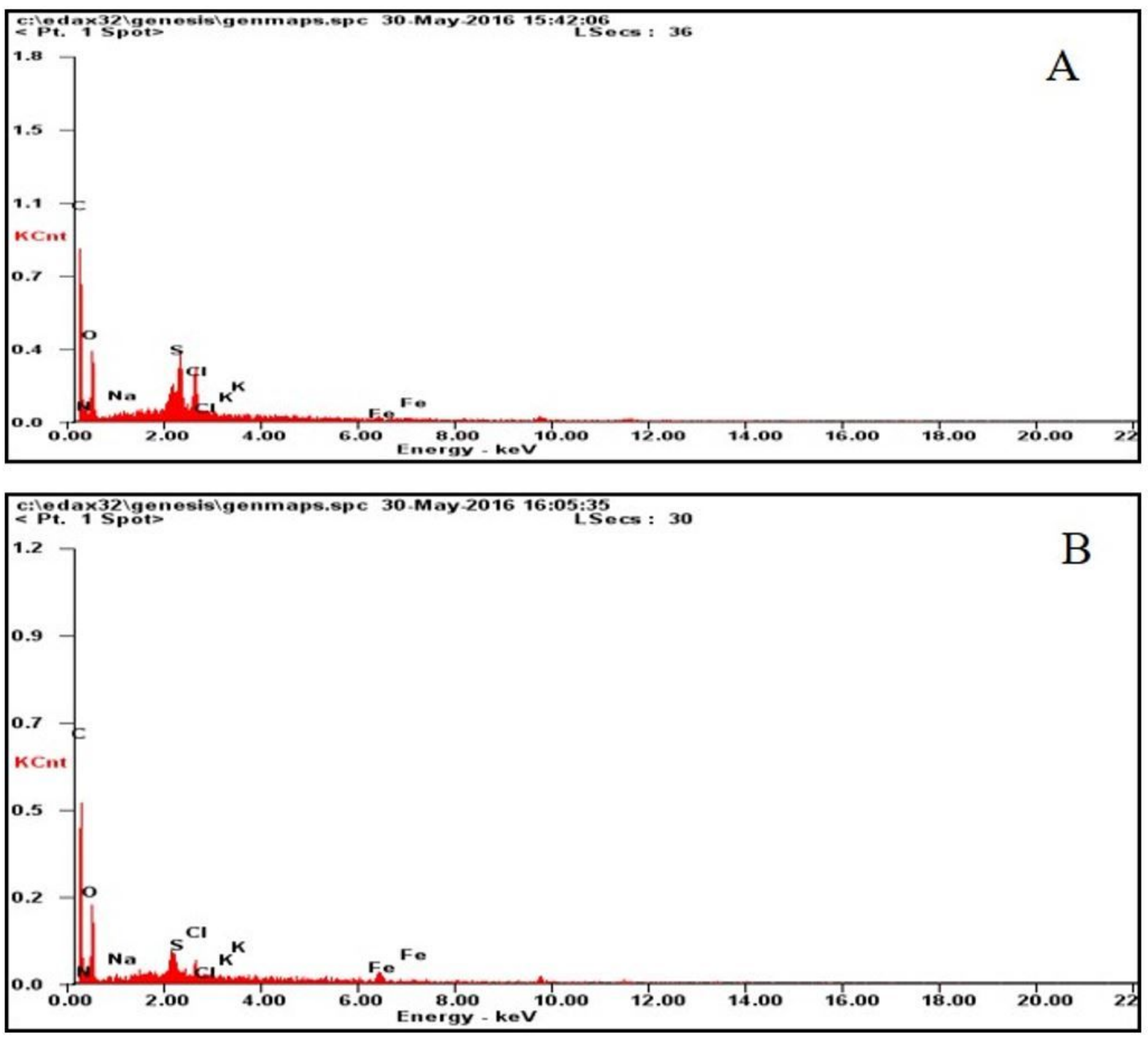

Figure 8

EDAX analysis of Extracellular melanin pigments from Prauserella sp.. (MAPPL 017A) A: Extracellular melanin; B: Synthetic melanin 

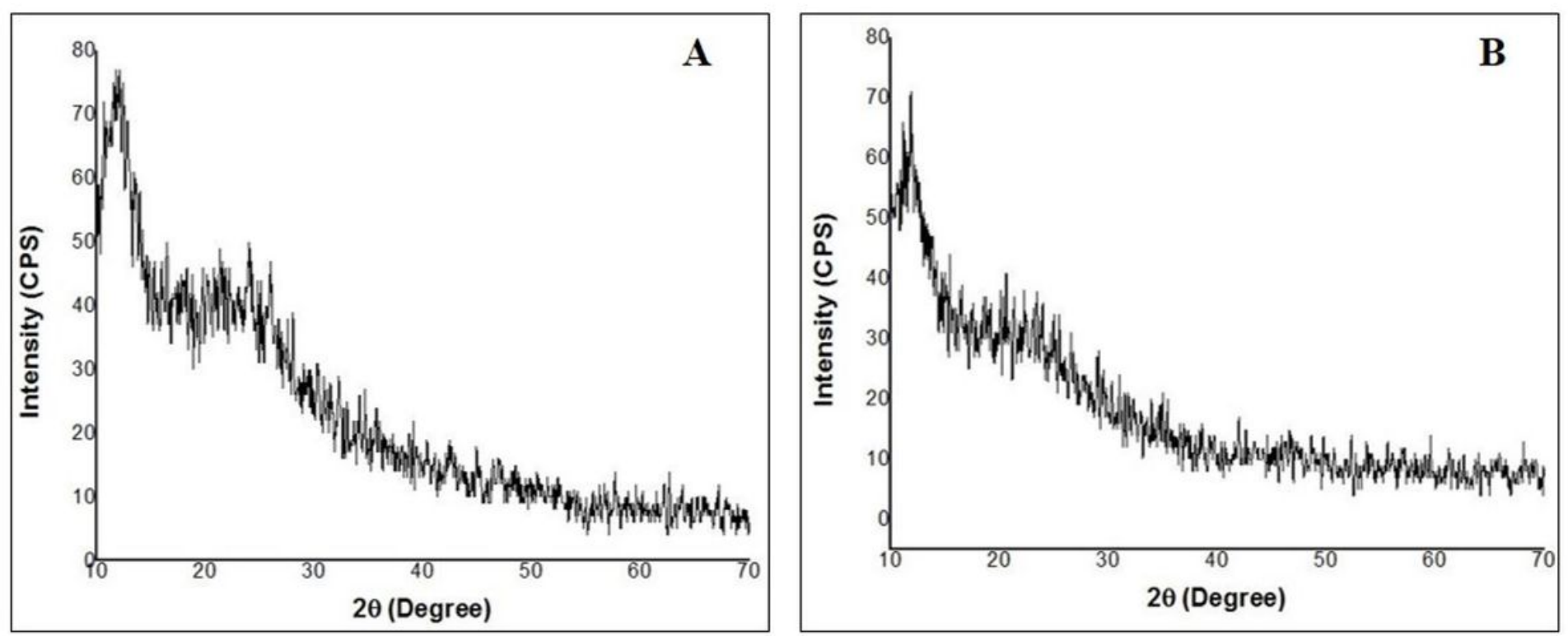

Figure 9

XRD pattern of the melanin pigments from Prauserella sp.. (MAPPL 017A) A. Extracellular melanin; B. Synthetic melanin 
Single Mass Analysis

Tolerance $=5.0 \mathrm{mDa} / \mathrm{DBE}: \min =-1.5, \max =50.0$

Element prediction: Off

Number of isotope peaks used for i-FIT $=3$

Monolsctople Mass, Even Electron lons

22 formula(e) evaluated with 1 results within limits (up to so best isotopic matches for each mass)

Elements Usod:

C: $0-18 \quad H: 0-10 \quad \mathrm{~N}: 0-2 \quad$ O: $0-4 \quad \mathrm{Na}: 0-1$

EXT-GANDHISHREE-EM

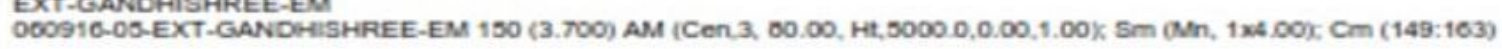

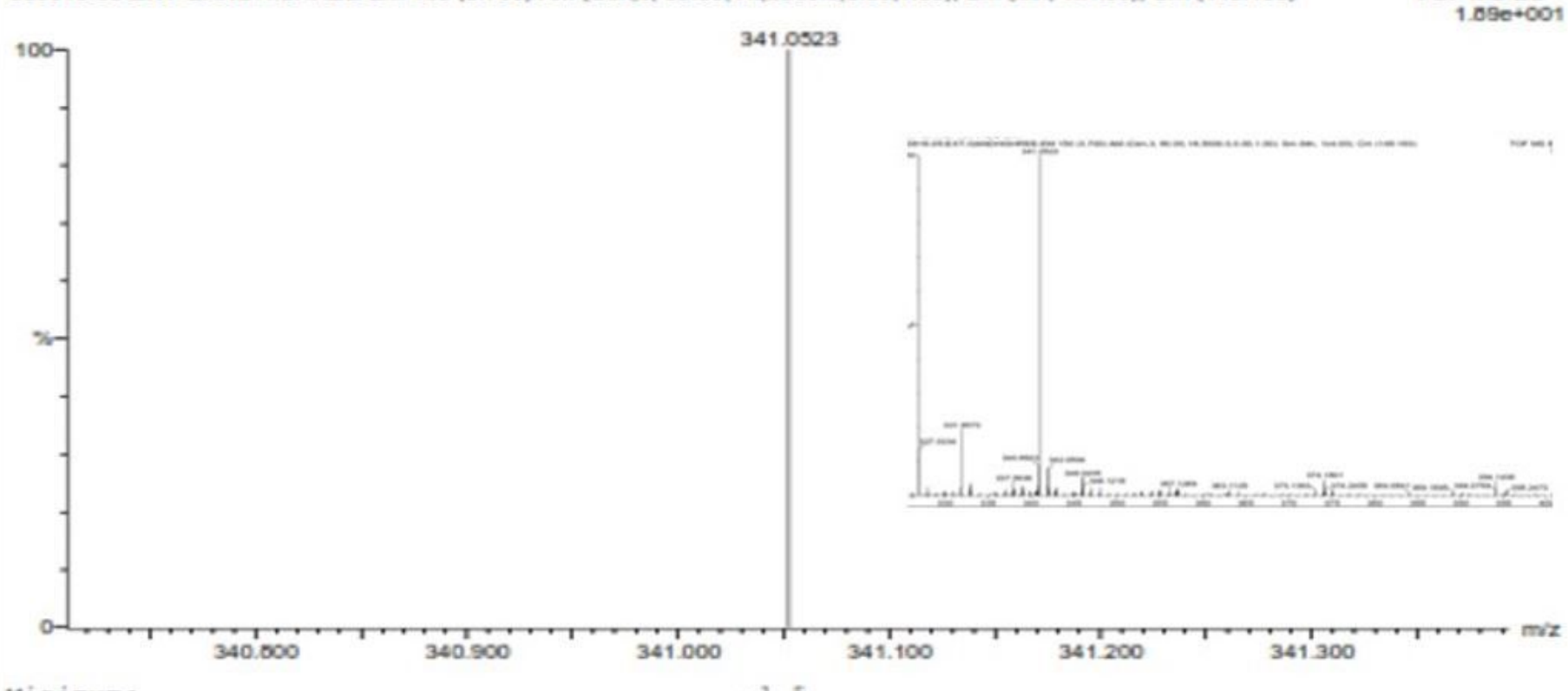

Minimum:

Maximum:

$\begin{array}{lll}5.0 & 10.0 \quad 50.5\end{array}$

Mass

Calc. Mass

nDa

PFM

DBE

i-Ft

Formula

$341.0523 \quad 342.0538$

$-1.5$

$-4.4$

14.5

$n / a$

Cl8 H1O $\mathrm{n2}$ O4 Na

Figure 10

ESI-MS analysis of extracellular melanin from Prauserella sp.. (MAPPL 017A) 

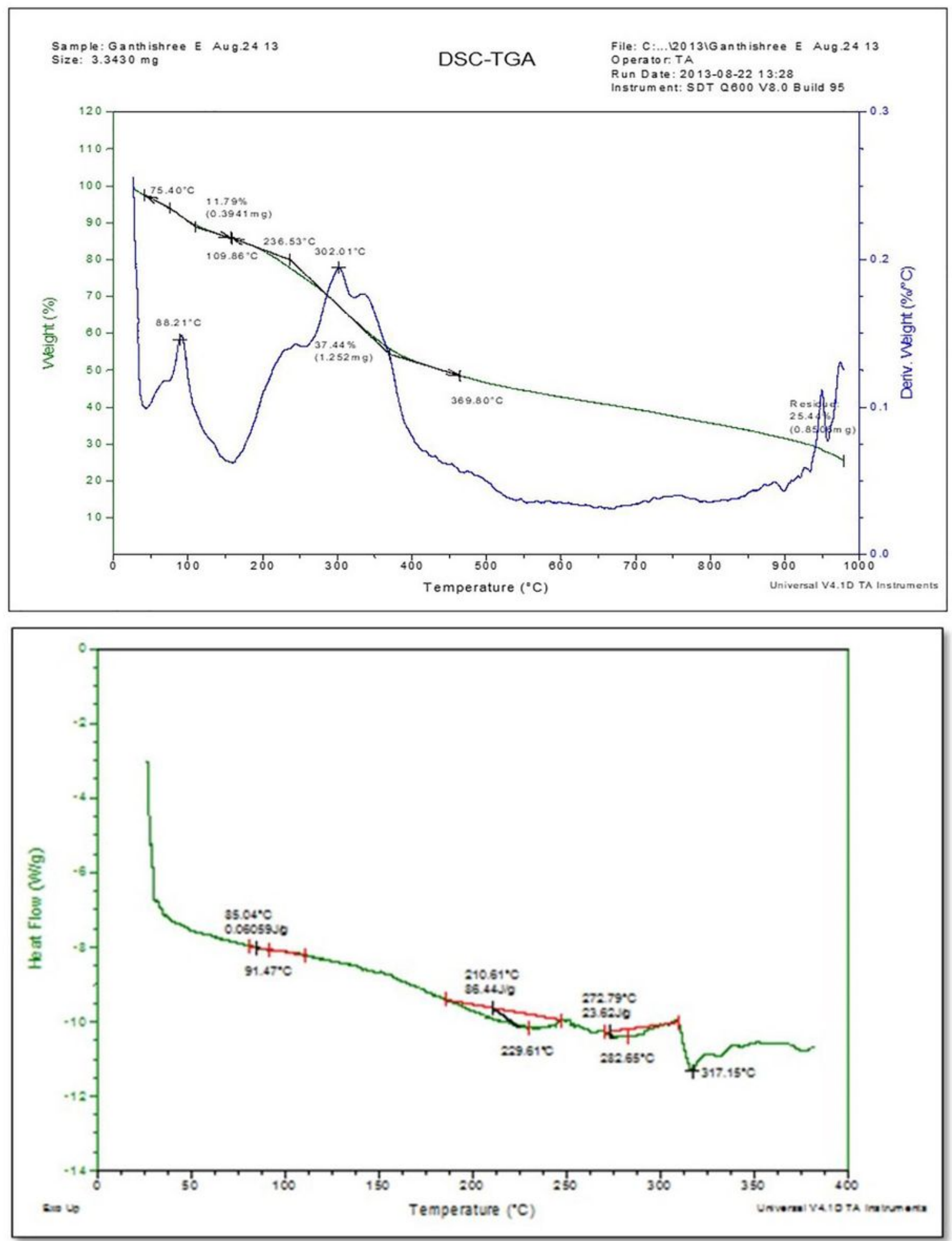

\section{Figure 11}

A. Thermal gravimetric analysis (TGA) of extracellular melanin from Prauserella sp.. (MAPPL 017A). B. Differential scanning colorimetric (DSC) analysis of extracellular melanin from Prauserella sp.. (MAPPL 017A). 